\title{
Aplicação do algoritmo harmony search no dimensionamento de perfis I soldados
}

\author{
Felipe Schaedler de Almeida ${ }^{1 *}$, Guilherme Dallagnol Vargas ${ }^{1}$ e Eduardo Braun ${ }^{1}$ \\ ${ }^{1}$ Departamento de Engenharia Civil, Universidade Federal do Rio Grande do \\ Sul,felipe.almeida@ufrgs.br,gui_dvargas@hotmail.com, \\ eduardo.braun@ufrgs.br
}

Application of harmony search algorithm in the design of welded I sections

\begin{abstract}
Resumo
Esse trabalho apresenta um estudo sobre a aplicação do algoritmo harmony search no dimensionamento de perfis I soldados submetidos à compressão, flexão ou à combinação desses esforços. A metodologia é desenvolvida para a automação do dimensionamento de elementos isolados, formulado como um problema de otimização não linear inteira-mista visando minimizar a função objetivo dada pela área da seção transversal. As espessuras da mesa e da alma são tomadas como variáveis discretas, limitadas pelos padrões de fabricação, enquanto a altura e a largura do perfil são tomadas como variáveis contínuas. Os critérios de segurança e desempenho da norma brasileira NBR8800:2008 são usados como restrições de projeto. Os exemplos mostram que o algoritmo é adequado ao projeto estrutural, pois tem bom desempenho em problems com variáveis mistas e espaço de resposta complexo.
\end{abstract}

Palavras-chave: Estruturas de aço, Otimização, Harmony search

\begin{abstract}
This work presents an investigation about the application of the harmony search algorithm in the design of welded steel I sections under compression, bending moment or both. The methodology is developed for the automation of the design of isolated elements, which is formulated as a mixed-integer nonlinear optimization problem aiming to minimize the objective function given by the cross section area. Flange and web thicknesses are taken as discrete design variables, restrained by the manufacturing standards, while the section height and width are taken as continuous variables. The safety and performance criteria given by the Brazilian standard NBR8800:2008 are used as design restraints. Examples show the good performance of the algorithm in the problems characterized by mixed variables and complex design space, indicating that the methodology is feasible for structural design.
\end{abstract}

Keywords: Steel structures, Optimization, Harmony search.

* autor correspondente 


\section{Introdução}

A metodologia tradicional do projeto estrutural se caracteriza por um processo iterativo onde uma solução proposta pelo projetista é avaliada quanto à sua segurança e desempenho, sendo posteriormente modificada para atingir os requisitos de projeto ou reduzir os custos de construção. Esse ciclo é interrompido muito mais pela limitação de tempo disponível para o projeto do que pelo esgotamento das possibilidades de aprimoramento da solução. A análise estrutural é uma das atividades de projeto que mais se beneficiou da introdução das técnicas computacionais. Hoje, a modelagem da estrutura é mais demorada do que a solução do problema estrutural e a determinação dos esforços nos elementos. O detalhamento da estrutura também teve seu tempo reduzido com a disseminação dos programas de CAD (computer aided design).

Por outro lado, a determinação das dimensões dos elementos que formam a estrutura continua sendo realizada pelo engenheiro com base em sua sensibilidade estrutural e nas informações obtidas no ciclo anterior do projeto. Encontrar a melhor solução para uma determinada estrutura é uma tarefa extremamente complexa, pois normalmente estão envolvidas muitas variáveis de projeto. Para auxiliar o desenvolvimento dessa etapa, existe um esforço para desenvolver metodologias que incorporam técnicas de otimização ao projeto estrutural. As características desse tipo de problema e os desafios associados são apresentados por Haftka e Gürda (1992).

A aplicação dos métodos e programação matemática aos problemas de otimização estrutural é difícil, pois os objetivos e as restrições são descritos por funções que costumam ser fortemente não lineares, descontínuas e não diferenciáveis. A presença simultânea de variáveis contínuas e discretas no dimensionamento estrutural também é um fator complicador para o sucesso dessas técnicas. Para contornar esses problemas, diversos métodos heurísticos têm sido usados na otimização estrutural.

O objetivo desse trabalho é estudar a aplicação do método heurístico harmony search (HS) na otimização de perfis I soldados de aço. A seção seguinte descreve brevemente o método. Posteriormente é discutida a formulação do problema de otimização e por fim são apresentados os exemplos de aplicação. Os resultados mostram que o algoritmo se 
adapta bem aos problemas de otimização não linear inteira-mista referentes ao dimensionamento dos perfis I soldados.

\section{0 algoritmo harmony search}

Harmony search é um algoritmo meta-heurístico desenvolvido por Geem et al. (2001) com inspiração no processo de improvisação que é comum em alguns ritmos musicais. As variáveis de projeto de um novo vetor de solução são copiadas de um conjunto das melhores soluções anteriores ou são definidas aleatoriamente, simulando experiência do músico com a melodia ou a improvisação, respectivamente. Adicionalmente, podem ser realizadas modificações sutis sobre os valores obtidos do conjunto das melhores respostas anteriores. Essa é uma operação com pequena chance de ocorrência, se equivalendo a um eventual ajuste da nota musical executada.

As soluções são representadas pelos vetores de harmonia (harmony vectors) $X=$ $\left\{x_{1}, x_{2}, x_{3}, \ldots x_{n}\right\}$, onde $n$ é o número de variáveis de projeto e $x_{i} \in\left[L_{i}, U_{i}\right]$ é o valor da variável de projeto $i$, que deve se enquadrar no intervalo definido pelos seus limites inferior e superior, $L_{i}$ e $U_{i}$, respectivamente. Conforme a otimização progride, as melhores soluções são colecionadas em uma memória de harmonias (harmony memory - HM), que é empregada na criação de novas soluções. O tamanho dessa memória é definida pelo parâmetro HMS (harmony memory size), que deve ser ajustado em cada processo de otimização. Lee e Geem (2005) descrevem o algoritmo HS com os seguintes passos:

Passo 1: Inicialização do algoritmo e do problema de otimização. Consiste em definir os parâmetros $H M S, H M C R, P A R$ e bw (definidos a seguir), que são empregados na criação de novos vetores solução pelo HS. Essa etapa também compreende a inicialização das rotinas de avaliação da função objetivo.

Passo 2: Inicialização da memória de harmonias (HM). São gerados vetores suficientes para preencher a HM tomando valores aleatórios para as variáveis.

Passo 3: Improvisação de novas harmonias. Cada variável de projeto $i$ de um novo vetor solução $\left(X^{\text {novo }}\right)$ pode ser definida por $x_{i}^{\text {novo }}=x_{i}^{j}$ cujo valor é obtido do vetor $X^{j}$ selecionado aleatoriamente na HM. Esse processo ocorre com uma probabilidade dada pela variável $H M C R \in[0,1]$ (harmony memory consideration rate). Alternativamente, há 
uma chance 1-HMCR do uso de um valor aleatório no intervalo admissível. O valor copiado da HM pode ser modificado através de uma operação denominada "pitch adjustment", cuja chance de ocorrência é dada pelo parâmetro PAR $\in[0,1]$ (pitch adjustment rate). Nesse caso, é aplicada uma perturbação sobre o valor original dentro de um intervalo definido pelo parâmetro bw (random distance bandwidth). Por fim, é avaliado o valor da função objetivo para $X^{\text {novo }}$.

Passo 4: Atualização da HM. O vetor criado $X^{\text {novo }}$ substitui a solução com maior valor da função objetivo na HM caso se configure uma solução melhor.

Passo 5: Verificação do critério de parada. A otimização é interrompida caso um critério de parada seja satisfeito, ou retorna ao passo 3 casos contrário. Nesse trabalho, a otimização é finalizada quando o número máximo de avaliações da função objetivo $\left(E_{\text {max }}\right)$ é atingido.

Além do algoritmo originalmente apresentado por Lee e Geem (2005) para a otimização de problemas de engenharia, vários aprimoramentos vêm sendo propostos a fim de tornar o método mais eficiente. Alia e Mandava (2011) apresentam uma ampla revisão sobre as diversas versões do HS. Degertekin (2008 e 2012) aplicou versões modificadas do HS à otimização de estruturas de aço e Kaveh e Abadi (2010) usaram esse algoritmo para a otimização de pisos em estruturas mistas.

Os estudos apresentados nesse artigo empregam a versão do HS dada por Almeida (2016). A principal modificação em relação ao algoritmo original está na variação do valor do parâmetro PAR ao longo da otimização e na forma de aplicação da operação "pitch adjustment". Também é incorporada a variação do parâmetro $b w$ a fim de enfatizar a exploração do espaço de respostas no início da otimização e o refinamento da busca em regiões promissoras no final da otimização.

\section{Formulação do problema de otimização de perfis I soldados}

De forma geral, o problema de otimização com restrições de desigualdade pode ser expresso por:

$$
\begin{gathered}
\text { Minimizar } f(X), \operatorname{com} X=\left\{x_{1}, x_{2}, \ldots x_{n}\right\}^{T} \\
\text { Sujeito a } g_{j}(X)<0 \text { e } L_{i} \leq x_{i} \leq U_{i}
\end{gathered}
$$


onde $f(X)$ é a função objetivo a ser minimizada e $X$ é o vetor com $n$ variáveis de projeto $\left(x_{i}\right)$. As funções $g_{j}(X)$ descrevem matematicamente cada uma das $m$ restrições do problema. Também costumam ser impostas restrições às variáveis de projeto, que devem se enquadrar no intervalo definido pelos limites inferior e superior $\left(L_{i}\right.$ e $\left.U_{i}\right)$ particulares a cada problema tratado. Como o HS é um método desenvolvido para minimização irrestrita, são empregadas funções de penalização para transformar as otimizações contendo restrições, como as estudadas nesse trabalho, em otimizações irrestritas. Nesse contexto, o problema a ser resolvido pelo HS passa a ser descrito por

$$
\text { Minimizar } f_{p}(X)=f(X)\left(1+\sum_{j=1}^{m} \beta_{j}\left(1+g_{j}\right)^{k_{j}} H\left(g_{j}\right)\right)
$$

onde $f_{p}(X)$ é a função objetivo com penalidades decorrentes das restrições definidas pelas funções $g_{j} . H\left(g_{j}\right)$ é a função de Heaviside (ou função degrau), assumindo valor zero para $g_{j}(X) \leq 0$ e valor 1 caso contrário. Os termos $\beta_{j}$ e $k_{j}$ controlam a intensidade da penalização pela violação da restrição $g_{j}$ e devem ser ajustados no início de cada problema de otimização. As restrições quanto aos valores máximos e mínimos assumidos pelas variáveis são automaticamente respeitados pelo processo de geração de novas soluções no HS.

O objetivo do presente trabalho é minimizar a quantidade de material necessário em uma barra formada por um perfil I soldado. São consideradas apenas barras isoladas submetidas a compressão simples, flexão em relação ao eixo de maior inércia ou à ação combinada desses esforços. As variáveis de projeto são a largura da mesa $\left(b_{f}\right)$ a altura total da seção $(h)$ e as espessuras das mesas e da alma, $t_{f}$ e $t_{w}$, respectivamente, conforme ilustrado na Fig. 1. Dessa forma, a função objetivo pode ser representada pela área total da seção transversal, sendo definida por

$$
f(X)=2 b_{f} t_{f}+\left(h-2 t_{f}\right) t_{\mathrm{w}}
$$

As variáveis $x_{1}$ e $x_{2}$ correspondem à altura e à largura do perfil, respectivamente, e são tratadas como variáveis contínuas com restrições $h_{\min }<x_{1}<h_{\max }$ e $b_{\min }<x_{2}<$ $b_{\max }$. Esses limites são definidos em cada problema de acordo com imposições arquitetônicas ou em função da experiência do projetista para uma dada situação. 


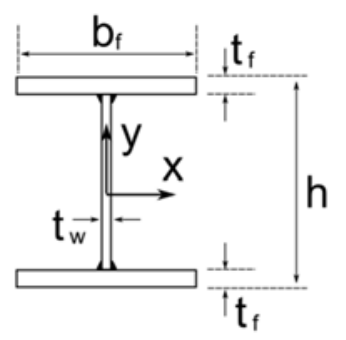

Figura 1 - Dimensões da seção transversal no perfil I soldado

Por outro lado, $x_{3}$ e $x_{4}$, que correspondem a $t_{w}$ e $t_{f}$, respectivamente, são tratadas como variáveis discretas, pois as espessuras das chapas usadas na fabricação dos perfis soldados são limitadas pela padronização adotada na indústria. Para adaptar o algoritmo a tal restrição, são empregadas codificações onde as espessuras disponíveis são associadas a números inteiros, conforme apresentado na Tabela 1. Dessa forma, as variáveis $x_{3}$ e $x_{4}$ assumem apenas valores inteiros que representam uma determinada espessura de chapa.

Tabela 1: Codificação das espessuras das chapas

\begin{tabular}{|c|c|c|c|c|c|c|c|c|c|c|}
\hline Código & 1 & 2 & 3 & 4 & 5 & 6 & 7 & 8 & 9 & 10 \\
\hline $\begin{array}{c}\text { Espessura } \\
(\mathrm{mm})\end{array}$ & 6,3 & 8,0 & 9,5 & 12,5 & 16 & 19 & 22,4 & 25 & 31,5 & 37,5 \\
\hline
\end{tabular}

\section{Exemplos de aplicação}

Nessa seção serão apresentados exemplos de aplicação do algoritmo HS na otimização de perfis I soldados. Esses exemplos têm como objetivo demonstrar que a metodologia proposta é capaz de gerar soluções otimizadas para elementos isolados submetidos a esforços de compressão, flexão ou à combinação dos dois. Um aço com módulo de elasticidade $E=200 \mathrm{GPa}$ e resistência ao escoamento $f_{y}=350 \mathrm{MPa}$ é empregado em todos os problemas discutidos a seguir. Os limites das variáveis são dados na Tabela 2 , a menos de $h_{\max }$, que é definido em cada exemplo.

Tabela 2: Limites inferior e superior das variáveis de projeto

\begin{tabular}{|c|c|c|}
\hline Variável & $L_{i}$ & $\boldsymbol{U}_{\boldsymbol{i}}$ \\
\hline $\begin{array}{ll}h & \left(x_{1}\right) \\
\end{array}$ & $100 \mathrm{~mm}$ & - \\
\hline$b_{f}\left(x_{2}\right)$ & $100 \mathrm{~mm}$ & $400 \mathrm{~mm}$ \\
\hline$t_{w}\left(x_{3}\right)$ & 1 & 7 \\
\hline$t_{f}\left(x_{4}\right)$ & 3 & 10 \\
\hline
\end{tabular}


As otimizações foram realizadas empregando memórias de tamanho $H M S=20$, com probabilidade de utilização definida por $H M C R=0,99$. A operação pitch adjutment é realizada empregando uma variação linear do parâmetro PAR, que decresce a partir do valor máximo inicial $P A R^{\prime}=0,30$ até o valor mínimo $P A R^{F}=0,10$ no fim da otimização. Para o random distance bandwidth é considerada uma variação exponencial, com o valor máximo no início, dado por $b w^{I}$, até o valor mínimo $b w^{F}$ no final da otimização. No caso das variáveis contínuas $\left(x_{1}\right.$ e $\left.x_{2}\right)$ é adotado $b w^{I}=50 \mathrm{~mm}$ e $b w^{F}=5 \mathrm{~mm}$. Para as variáveis discretas $\left(x_{3}\right.$ e $\left.x_{4}\right)$ são assumidos os valores $b w^{I}=3$ e $b w^{F}=1$.

A otimização é finalizada quando o número de avaliações da função objetivo $(E)$ atinge o limite $E_{\max }=1000$, adotado em todos os casos. Com esse número de soluções avaliadas, cada otimização é realizada em uma fração de segundo pelo código implementado em linguagem Fortran 2003 e executado em um processador moderno (Intel i5-3570). Em princípio, poderia ser adotado um valor $E_{\max }$ muitas vezes maior, melhorando o desempenho do algoritmo sem tornar o tempo de otimização inviável. A limitação ao valor de $E_{\max }$ adotado visa criar condições para testes similares às que se verificariam em um projeto onde os recursos computacionais seriam divididos para a otimização das muitas barras que compõem a estrutura.

Como o HS é um algoritmo baseado em operações randômicas, o resultado final pode ser diferente em cada processo de otimização. Nesse estudo foram realizadas 100 otimizações em cada problema, o que torna possível identificar a qualidade da solução e sua evolução com as iterações. Isso permite a caracterização do desempenho do HS no tipo de problema estudado.

\subsection{Compressão simples}

Esse exemplo apresenta a aplicação da metodologia à otimização de uma barra submetida à compressão simples. São adotadas duas restrições para essa condição de carregamento. A primeira se refere à resistência à compressão, sendo definida por

$$
g_{1}(X)=\frac{N_{c, S d}}{N_{c, R d}(X)}-1
$$

onde $N_{c, S d}$ é a força axial de compressão solicitante de cálculo e $N_{c, R d}$ é a força axial de compressão resistente de cálculo, determinada em função das variáveis de projeto $(X)$. 
A força $N_{c, S d}$ é obtida pela combinação dos carregamentos atuando na estrutura multiplicados pelos devidos coeficientes de majoração e informada como dado de entrada na otimização. A resistência é calculada para cada solução gerada usando os critérios definidos na NBR8800:2008 (ABNT, 2008). A penalização pela violação dessa restrição é aplicada com os coeficientes $\beta_{1}=1$ e $k_{1}=2$.

A segunda restrição se refere à esbeltez máxima de 200 que é admitida para a barra comprimida na NBR8800:2008. Tal restrição é definida por

$$
g_{2}(X)=\frac{\max \left(\frac{k_{x} L_{x}}{r_{x}}, \frac{k_{y} L_{y}}{r_{y}}\right)}{200}-1
$$

onde $k_{x} L_{x}$ e $k_{y} L_{y}$ são os comprimentos de flambagem por flexão em torno dos eixos principais centrais $x$ e $y$ da seção, respectivamente, e $r_{x}$ e $r_{y}$ são os raios de giração da seção em torno dos mesmos eixos. A penalização devida à violação dessa restrição é aplicada com coeficientes $\beta_{2}=1000$ e $k_{2}=2$.

Nesse estudo é considerada uma barra de comprimento $L=5 \mathrm{~m}$ com as duas extremidades rotuladas $\left(k_{x} L_{x}=k_{y} L_{y}=k_{z} L_{z}=L\right)$ e a altura máxima da seção é fixada em $h_{\max }=400 \mathrm{~mm}$. O primeiro teste foi realizado para uma força solicitante $N_{c, S d}=$ $5500 \mathrm{kN}$. Entre as 100 otimizações realizadas, o melhor resultado obtido pelo HS apresentou área $A=20225 \mathrm{~mm}^{2}$ e força resistente $N_{c, R d}=5500,02 \mathrm{kN}$, com as variáveis de projeto assumindo os seguintes valores: $h=287,4 \mathrm{~mm}, b_{f}=400 \mathrm{~mm}$, $t_{w}=9,5 \mathrm{~mm}$ e $t_{f}=22,4 \mathrm{~mm}$. A Fig. 2 mostra como o valor médio, mínimo e máximo da área da solução ótima $\left(A_{o t}\right)$ evolui em função do número de avaliações da função objetivo realizadas pelo HS ao longo da otimização. 


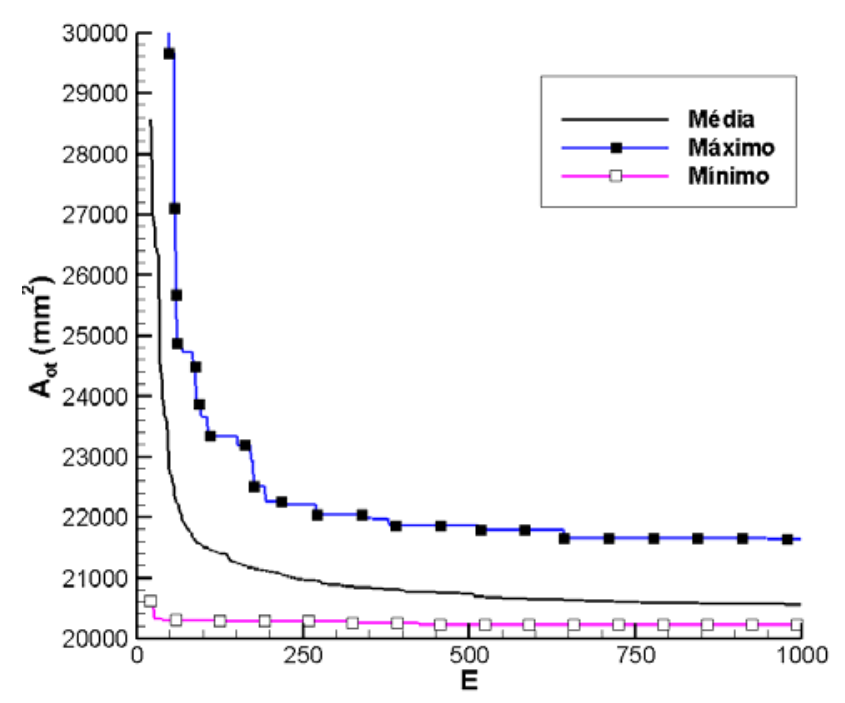

Figura 2 - Área mínima na otimização $\left(N_{c, S d}=5500 \mathrm{kN}\right)$

No estudo seguinte foram realizadas otimizações considerando a intensidade da força solicitante $\left(N_{c, S d}\right)$ entre $3000 \mathrm{kN}$ e $8500 \mathrm{kN}$ (com valores tomados em intervalos de 500 kN). A Fig. 3 apresenta a área da seção transversal otimizada em função da força de compressão considerada. Se observa uma relação aproximadamente linear com variação de $3,48 \mathrm{~mm}^{2} / \mathrm{kN}$. A relação $N_{C, R d} \times A$ das seções CS400×106 a CS400x248 da série CS padronizada pela NBR5884:2013 são apresentadas na mesma figura para fins de comparação. Os resultados mostram que o algoritmo de otimização sempre foi capaz de gerar soluções com área ligeiramente menor que as seções padronizadas.

A figura 4 apresenta a variação das dimensões da seção transversal obtida nas otimizações considerando forças de compressão entre 3000 kN e 8500 kN. As dimensões das mesas $\left(b_{f}\right.$ e $\left.t_{f}\right)$ têm grande influência na otimização. A espessura da mesa sempre cresce com o aumento da força normal. A largura da mesa converge rapidamente ao limite superior de $400 \mathrm{~mm}$, apresentando pequenas reduções quando $t_{f}$ aumenta. De forma semelhante, a altura total da seção cresce quando $t_{f}$ é constante e diminui quando $t_{f}$ aumenta, mas nunca atinge o limite superior $h_{\max }=400 \mathrm{~mm}$. A espessura $t_{w}$ oscila em torno dos menores valores, mostrando a importância relativamente pequena da alma na resistência do perfil. 


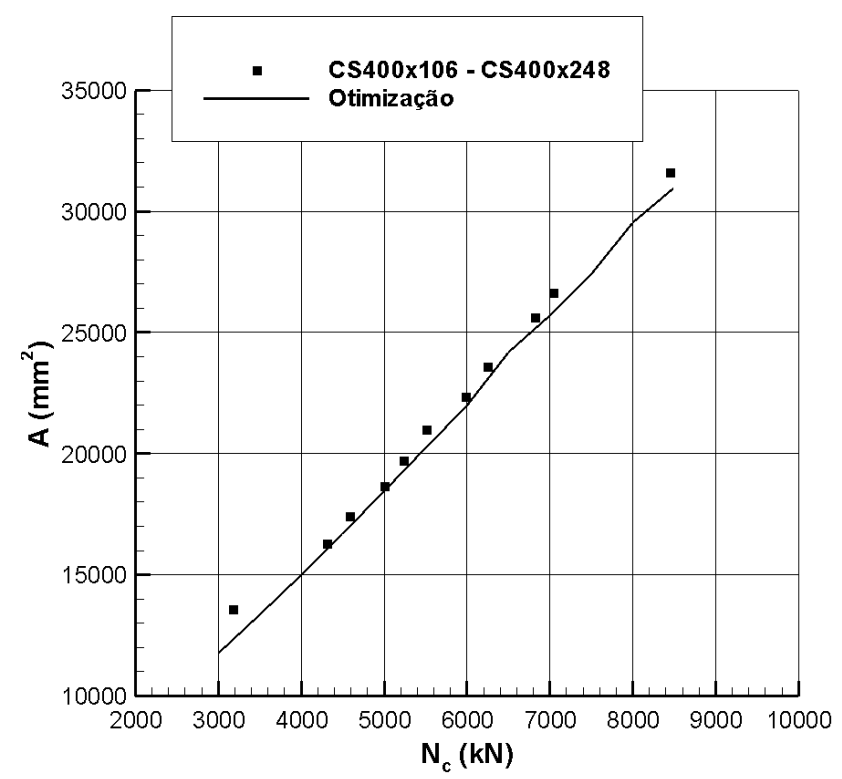

Figura 3 - Área ótima em função da força normal
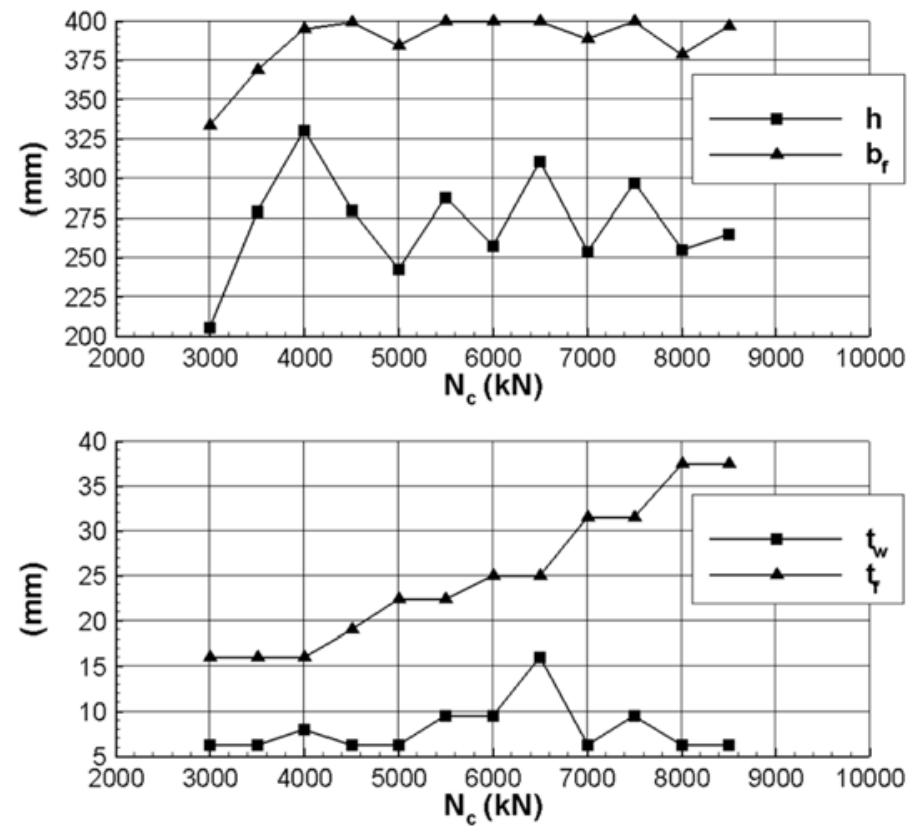

Figura 4 - Dimensões da seção otimizada em função da força normal

\subsection{Flexão simples}

Nesse exemplo é tratado o problema de otimização de uma viga simplesmente apoiada e submetida a um carregamento transversal uniformemente distribuído ao longo de todo o vão, conforme a Fig. 5 ilustra. 


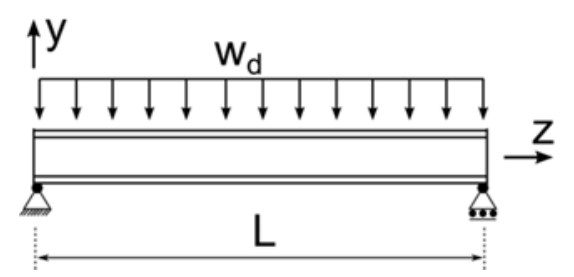

Figura 5 - Viga simplesmente apoiada submetida a carga uniformemente distribuída A minimização da área da seção transversal é realizada considerando cinco restrições. Primeiramente, a viga deve atender às condições de segurança quanto ao momento fletor, conforme as prescrições da NBR8800:2008. Essas restrições são definidas por:

$$
\begin{gathered}
g_{1}(X)=\frac{M_{S d}}{M_{R d}^{F L M}(X)}-1, \quad g_{2}(X)=\frac{M_{S d}}{M_{R d}^{F L A}(X)}-1, \quad g_{3}(X) \\
=\frac{M_{S d}}{M_{R d}^{F L T}(X)}-1
\end{gathered}
$$

Nessas equações, $M_{S d}$ é o momento fletor solicitante de cálculo, obtido em função da intensidade da carga distribuída $\left(W_{d}\right)$ e do vão da viga $(L)$. Os termos $M_{R d}^{F L M}, M_{R d}^{F L A}$ e $M_{R d}^{F L T}$ se referem ao momento resistente para os estados limites de flambagem local da mesa (FLM), flambagem local da alma (FLA) e flambagem lateral com torção (FLT), respectivamente. Nas avaliações relacionadas à FLT, foi considerado o fator de modificação para diagrama de momento fletor não uniforme com valor $C_{b}=1,136$, que corresponde à distribuição de momento nas condições do problema. Também foi considerada a limitação a vigas de alma não esbelta, o que é garantido pela função de restrição

$$
g_{4}(X)=\frac{\left(h-2 t_{f}\right) / t_{0}}{5,7 \sqrt{E / f_{y}}}-1
$$

A última restrição está relacionada ao deslocamento transversal excessivo, que muitas vezes é a condição determinante no projeto de vigas de aço. Para atender essas condições, é imposto um limite inferior para o momento de inércia da viga. A Eq. (8) descreve essa restrição, onde foi introduzido o momento de inércia mínimo $\left(I_{\min }\right)$ para que o deslocamento da viga tratada no problema não exceda um valor admissível $\delta_{a d m}=L / \alpha$. 


$$
g_{5}(X)=\frac{I_{\min }}{I_{x}(X)}-1, \quad \text { com } \quad I_{\min }=\frac{5}{384} \frac{\alpha W_{d} L^{3}}{E}
$$

No problema estudado é adotado o comprimento $L=6 \mathrm{~m}$ e o deslocamento transversal admissível é dado por $\alpha=350$. São considerados os casos de vigas com altura máxima $h_{\max }=400 \mathrm{~mm}$ e $h_{\max }=600 \mathrm{~mm}$. Os coeficientes para penalização por violação das restrições são $\beta_{i}=1(i=1,2,3,5), \beta_{4}=100$ e $k_{i}=2(i=1-5)$. As otimizações são realizadas para carregamentos com intensidade $W_{d}$ variando de 10 $\mathrm{kN} / \mathrm{m}$ a $100 \mathrm{kN} / \mathrm{m}$ (em intervalos de $10 \mathrm{kN} / \mathrm{m}$ ).

A Fig. 6 (a) apresenta a variação da área $(A)$ da seção otimizada em função da carga distribuída atuando sobre a viga. O comportamento é idêntico para os dois casos estudados até a intensidade de $30 \mathrm{kN} / \mathrm{m}$. A partir desse ponto a limitação de altura $h_{\max }=400 \mathrm{~mm}$ se torna ativa e ocorre a diferenciação das soluções ótimas. A área cresce lentamente com o aumento de $W_{d}$ para o caso com $h_{\max }=600 \mathrm{~mm}$ enquanto que um crescimento linear a uma taxa de aproximadamente $33 \mathrm{~mm}^{2} /(\mathrm{kN} / \mathrm{m})$ é observado na viga com altura restringida. É importante ressaltar que as soluções ótimas apresentam as restrições 3 e 5 simultaneamente ativas ou muito próximas da ativação. Uma abordagem mais geral sobre o dimensionamento de vigas também deveria incluir restrição quanto à força cortante. Especificamente no problema apresentado, as seções transversais otimizadas sempre apresentam uma força cortante correspondente à plastificação da alma $\left(V_{p l}\right)$ suficientemente alta para que a resistência ao cisalhamento seja atendida com uso de enrijecedores transversais, se necessários.

A menos da espessura da alma, que se mantém no valor mínimo $t_{w}=6,3 \mathrm{~mm}$, a diferença da variação das dimensões da seção transversal nos dois casos também é bem evidente, como mostra a Fig. 6 (b). No caso com $h_{\max }=600 \mathrm{~mm}$, a altura e a largura total aumentam suavemente com a espessura da mesa constante $t_{f}=9,5 \mathrm{~mm}$. No outro caso, a altura fica limitada a $400 \mathrm{~mm}$ a partir de $40 \mathrm{kN} / \mathrm{m}$, gerando um forte aumento da largura $b_{f}$ com espessura constante $t_{f}=9,5 \mathrm{~mm}$. Esse comportamento se estende até a carga de $60 \mathrm{kN} / \mathrm{m}$, a partir da qual a espessura da mesa também passa a aumentar. Nessa fase, a largura $b_{f}$ oscilar a cada incremento de $t_{f}$. 


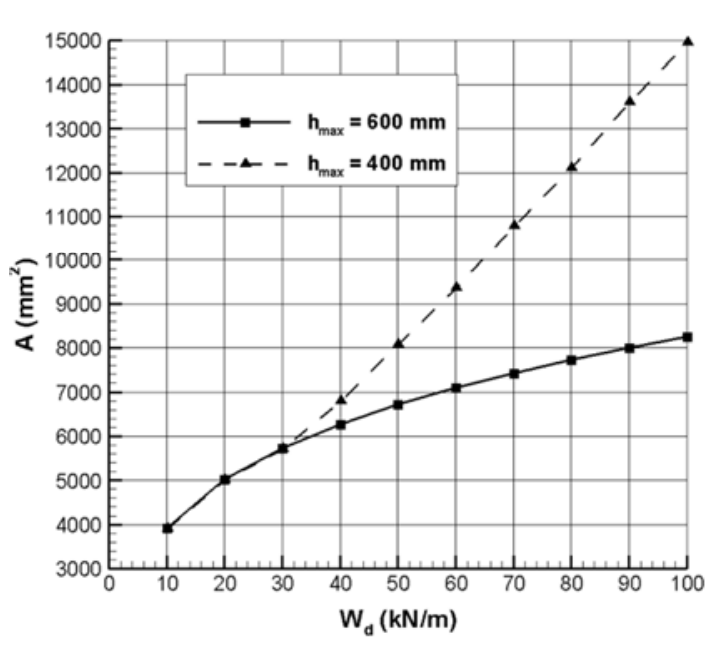

(a)
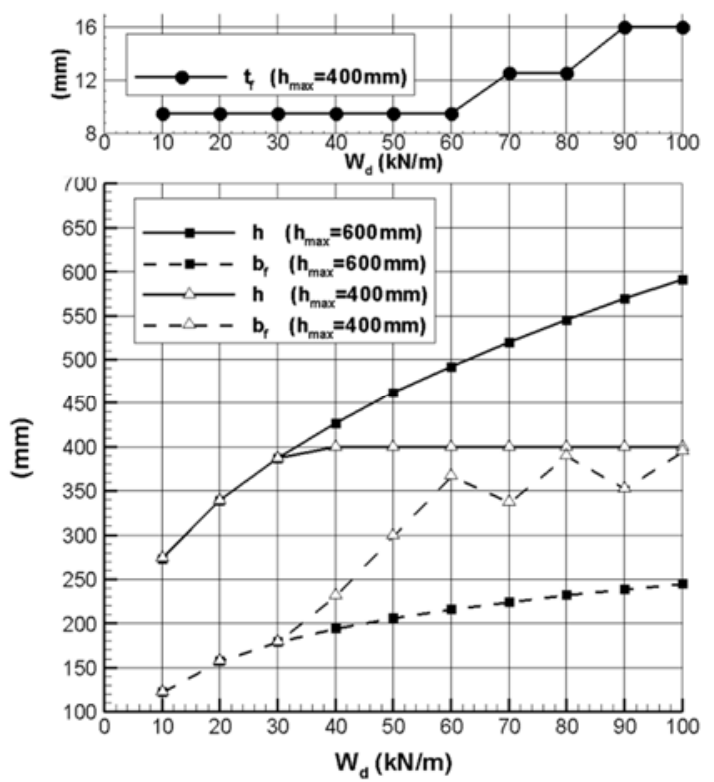

(b)

Figura 6 - Área e dimensões da seção otimizada em função de $W_{d}$

\subsection{Compressão e flexão combinadas}

Esse último exemplo apresenta um estudo sobre a aplicação da metodologia de otimização ao dimensionamento de perfis I soldados submetidos a compressão e flexão combinadas. A Fig. 7 ilustra a situação considerada, com os momentos $M_{1}$ e $M_{2}$ atuando em conjunto com a força de compressão $N_{c}$ nas extremidades. Os resultados são obtidos para condições semelhantes às do exemplo na seção $4.1, \operatorname{com} L=5 \mathrm{~m}$, ligações rotuladas $\left(k_{x} L_{x}=k_{y} L_{y}=k_{z} L_{z}=L\right)$ e a limitação $h_{\max }=400 \mathrm{~mm}$. São obtidas soluções ótimas para o caso com momentos iguais provocando curvatura simples $\left(M_{1}=M_{2}=M\right)$, com magnitude proporcional à carga axial dado por $M=e N_{c}$.

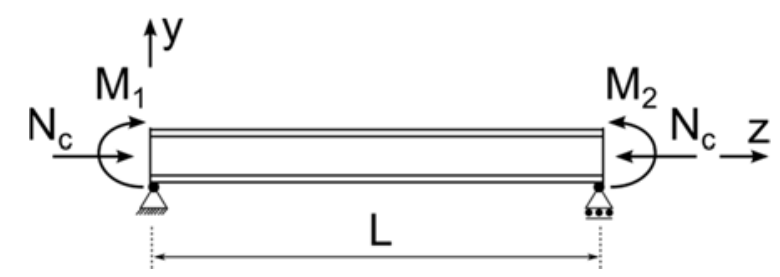

Figura 7 - Barra submetida a compressão e flexão

A restrição adotada em função da segurança da barra quanto aos esforços combinados é expressa por 


$$
g_{1}(X)=f_{\text {int }}-1
$$

A função de interação $\left(f_{\text {int }}\right)$ é definida pela NBR8800:2008 e pode ser escrita no contexto desse trabalho como

$$
\begin{gathered}
f_{\text {int }}=\frac{N_{S d}}{2 N_{R d}}+\frac{M_{S d}}{M_{R d}}, \text { para } \frac{N_{S d}}{N_{R d}} \leq 0,2 \\
f_{\text {int }}=\frac{N_{S d}}{N_{R d}}+\frac{8}{9} \frac{M_{S d}}{M_{R d}}, \text { para } \frac{N_{S d}}{N_{R d}}>0,2
\end{gathered}
$$

onde $N_{c, R d}$ é a resistência da barra à compressão simples e $M_{R d}$ é a resistência da barra à flexão em torno do eixo $x$, tomada como o menor valor entre $M_{R d}^{F L M}, M_{R d}^{F L A}$ e $M_{R d}^{F L T}$ (com o coeficiente $C_{b}=1$ em decorrência do momento constante). Os coeficientes de penalização para essa restrição forma tomados com os valores $\beta_{1}=1$ e $k_{1}=2$.

Os efeitos de segunda ordem devidos à força axial são considerados na determinação do momento solicitante por $M_{S d}=B_{1} M$, onde o termo $B_{1}$ é calculado pela seguinte equação (ver anexo D da NBR8800:2008):

$$
B_{1}=\frac{C_{m}}{1-\frac{N_{S d}}{N_{e}}} \geq 1
$$

Considerando as características do problema analisado, $C_{m}=1$ e $N_{e}$ se refere à carga de flambagem elástica por flexão em torno do eixo $x$. Além da restrição quanto à segurança definida pela Eq. (9), também são consideradas restrições relativas à esbeltez global máxima de 200 para a barra e à esbeltez máxima da alma, definidas pelas equações (5) e (7), respectivamente.

A Fig. 8 mostra a variação da área da seção transversal do perfil otimizado considerando a mesma faixa de força axial empregada na seção 4.1 (3000 kN $\leq N_{c} \leq 8500 \mathrm{kN}$ ) e três relações entre $M$ e $N_{c}$ dados por $e$ igual a $20 \mathrm{~mm}, 40 \mathrm{~mm}$ e $60 \mathrm{~mm}$. Para fins de comparação, também estão incluídos os resultados correspondentes à compressão simples $(e=0 \mathrm{~mm}$ ) que foram obtidos no primeiro exemplo. A relação praticamente linear entre a área ótima e a força de compressão é observada para todos os casos estudados, seguindo a mesma tendência verificada para a compressão simples. Como esperado, a área ótima é maior para os casos com momento de maior magnitude (definido pelo valor de $e$ ) e as curvas apresentam afastamento crescente à medida que 
$N_{c}$ aumenta. As curvas correspondentes a $e$ igual a $40 \mathrm{~mm}$ e $60 \mathrm{~mm}$ são interrompidas em $N_{c}$ igual a $8000 \mathrm{kN}$ e $7500 \mathrm{kN}$, respectivamente, pois não há soluções dentro dos limites das variáveis que atendam à restrição estabelecida pela Eq. (9).

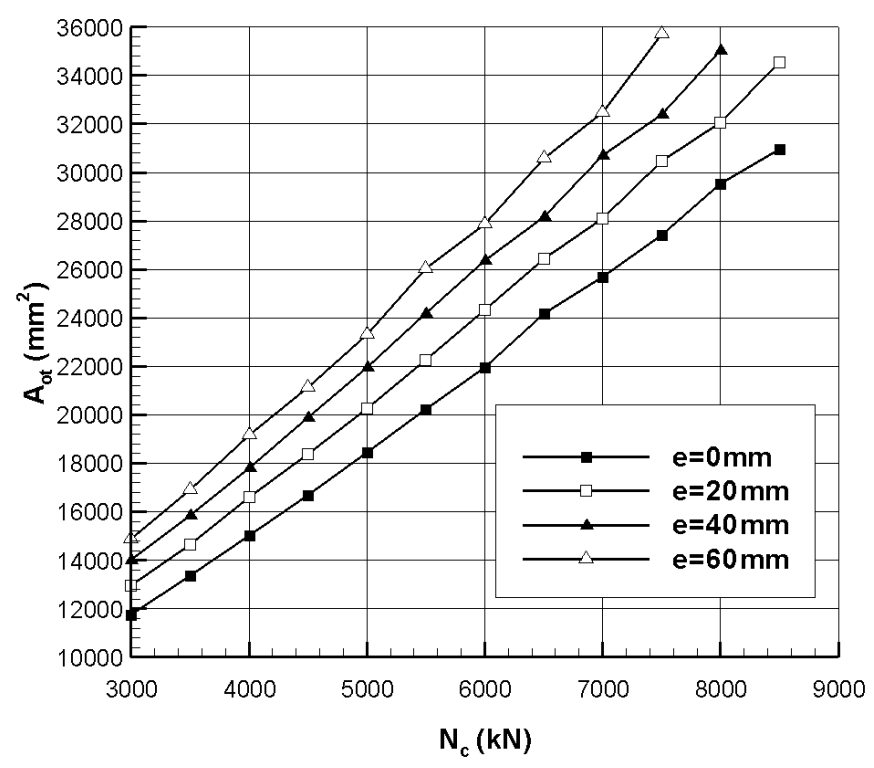

Figura 8 - Área da seção otimizada em função de $N_{c}$

A Fig. (9) mostra a variação das dimensões da seção transversal ótima em função da força axial para os diversos casos da relação $e=M / N_{c}$. A altura do perfil otimizado tende cada vez mais rápido ao limite superior $h_{\max }=400 \mathrm{~mm}$ à medida que maiores valores de $e$ são considerados, refletindo a maior exigência de inércia e resistência em relação ao eixo de flexão nesses casos. Um efeito semelhante é observado para a espessura da mesa $\left(t_{f}\right)$. Por outro lado, a espessura da alma $\left(t_{w}\right)$ se mantém em valores relativamente baixos, o que indica sua pequena influência sobre a resistência do elemento submetido aos esforços combinados. Assim como nos exemplos anteriores, se verifica um leve decréscimo das variáveis contínuas sempre que a variável discreta de maior relevância no problema $\left(t_{f}\right)$ sofre um incremento. 

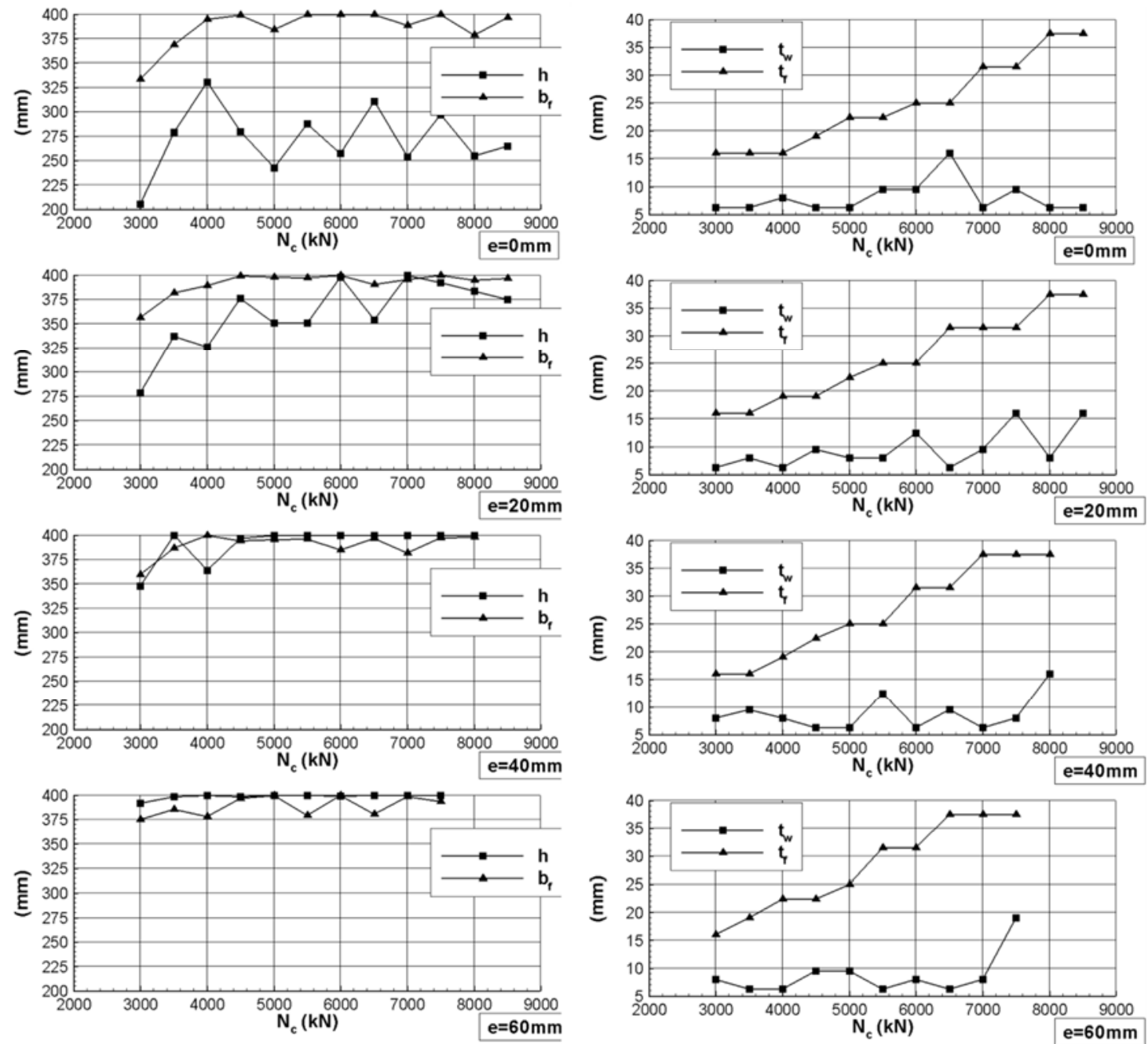

Figura 9 - Dimensões da seção otimizada em função de $N_{c}$

\section{Conclusão}

A metodologia de projeto baseada em otimização que foi proposta nesse trabalho se mostrou adequada para tratar os problemas analisados. De forma geral, o algoritmo HS é capaz de lidar com o espaço de resposta complexo decorrente das restrições impostas no projeto de perfis soldados, além de acomodar simultaneamente variáveis discretas e contínuas sem maiores dificuldades, gerando soluções muito competitivas.

Embora os casos estudados não reflitam necessariamente situações práticas encontradas em estruturas reais, eles servem como exercício acadêmico para demonstrar as potencialidades do método e o comportamento geral esperado para as soluções. Os casos específicos de projeto, com diferentes restrições construtivas ou econômicas, podem ser facilmente contemplados com a formulação apresentada nesse estudo. 
Os trabalhos futuros devem considerar a otimização dos elementos que compõem um sistema estrutural, levando em conta a influência das características das barras sobre o comportamento global da estrutura. Também devem ser exploradas formulações alternativas para o problema de otimização onde a função objetivo é definida pelo preço final da estrutura, pois essa é uma medida de eficiência mais representativa do que a quantidade de aço utilizado.

\section{Agradecimentos}

Os autores agradecem ao Conselho Nacional de Desenvolvimento Científico e Tecnológico (CNPq) e à Coordenação de Aperfeiçoamento de Pessoal de Nível Superior (Capes) pelo apoio às pesquisas divulgadas nesse artigo.

\section{Referências bibliográficas}

Alia, O., Mandava, R. The variants of the harmony search algorithm: an overview. Artificial Intelligence Review. v. 36, n. 1, p. 49-68, 2011

Almeida, F.S. Stacking sequence optimization for maximum buckling load of composite plates using harmony search algorithm, Composite Structures, v. 143, n. 20, p.287-299, 2016

ASSOCIAÇÃO BRASILEIRA DE NORMAS TÉCNICAS. NBR8800:2008 - Projeto de estruturas de aço e de estruturas mistas de aço e concreto de edifícios, 2008

ASSOCIAÇÃO BRASILEIRA DE NORMAS TÉCNICAS. NBR5884:2013 - Perfil I estrutural de aço soldado por arco elétrico - Requisitos gerais, 2013

Degertekin, S.O. Optimum design of steel frames using harmony search algorithm, Structural and Multidisciplinary Optimization v. 36, n. 4, p. 393-401, 2008

Degertekin, S.O. Improved harmony search algorithms for sizing optimization of truss structures, Computers \& Structures v. 92-93, p. $229-241,2012$

Geem, Z.W., Kim, J.H., Loganathan, G.V. A new heuristic optimization algorithm: harmony search. Simulation. v. 76, n. 2, p. 60-68, 2001

Haftka, R. T., Gürdal, Z. Elements of Structural Optimization. Springer, Netherlands, 1992

Lee, K., Geem, Z. A new meta-heuristic algorithm for continuous engineering optimization: Harmony search theory and practice. Computer Methods in Applied Mechanics and Engineering, v. 194, p. 3902-3933, 2005

Kaveh, A., Abadi, A.S.M. Cost optimization of a composite floor system using an improved harmony search algorithm, Journal of Constructional Steel Research, v. 66, n. 5, p. $664-669$, 2010 